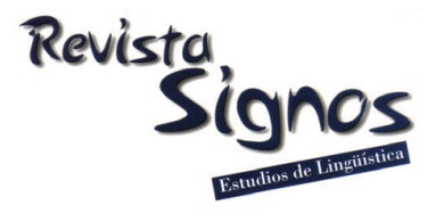

\title{
El deterioro de la fluidez verbal como marcador de déficit comunicativo primario en el primer episodio de Esquizofrenia
}

\author{
The evaluation of verbal fluency as a marker of primary \\ communicative deficit in the first episode of schizophrenia
}

Alicia Figueroa
Barra
UniversidAD DE ChILE
CHILE
aliciafigueroa@med.uchile.cl

\author{
Nieves Mendizábal \\ De La Cruz \\ UNIVERSIDAD DE VALLADOLID \\ ESPAÑA \\ marianieves.mendizabal@uva.es
}

\author{
Natalia Jimeno \\ Bulnes \\ UNIVERSIDAD DE VALLADOLID \\ ESPAÑA \\ najimeno@med.uva.es
}

Recibido: 21-III-2018 / Aceptado: 22-X-2018

DOI: $10.4067 /$ S0718-09342019000300780

\section{Resumen}

En este trabajo se analizan algunas dimensiones de la fluidez verbal en una muestra de 50 entrevistas clínicas realizadas con personas diagnosticadas con Esquizofrenia crónica y primer episodio, desde la perspectiva de la lingüística clínica aplicada a la psiquiatría. Nos proponemos determinar si existen diferencias en las dimensiones de fluidez verbal estudiadas entre ambos grupos de pacientes. De esta forma, para evaluar si constituye un marcador de déficit lingüístico primario, pretendemos verificar la relación entre la fluidez verbal y la etapa de evolución la enfermedad. El análisis de los datos, en primer lugar, demuestra que es posible observar el deterioro temprano de la fluidez verbal en personas con primer episodio de Esquizofrenia. En el segundo caso, la etapa clínica variable de la enfermedad presenta una mayor significación estadística, por lo tanto, es posible considerar que el deterioro de la fluidez verbal es un marcador primario del déficit pragmalingüístico para la Esquizofrenia.

Palabras Clave: Fluidez verbal, Esquizofrenia, primer episodio, marcador primario, lingüística clínica. 


\begin{abstract}
This paper analyzes some dimensions of verbal fluency in a sample of 50 clinical interviews conducted with people diagnosed with chronic schizophrenia and first episode, from the perspective of clinical linguistics applied to psychiatry. We propose to determine if there are differences in the verbal fluidity dimensions studied between both groups of patients. In this way, in order to assess whether it constitutes a marker of primary linguistic deficit, we intend to verify the relationship between verbal fluency, clinical diagnosis and the stage of the disease. Firstly, the analysis of the data shows that it is possible to observe the early deterioration of verbal fluency in people with first episode of schizophrenia. In the second place, the variable clinical stage of the disease presents a greater statistical significance; therefore, it is possible to consider that the deterioration of verbal fluency is a primary marker of the pragmalinguistic deficit for schizophrenia.
\end{abstract}

Key Words: Verbal fluency, schizophrenia, first episode, primary marker, clinical linguistics.

\title{
INTRODUCCIÓN
}

La fluidez verbal (FV) es una compleja dimensión de la comunicación debido a que está constituida por diversos componentes y factores cognitivos. En su funcionamiento intervienen una serie de procesos cerebrales y mentales que condicionan la interacción entre los hablantes. Las fallas en la FV se asocian al deterioro en el córtex frontal que es una de las características propias de las enfermedades neurodegenerativas y de patologías psiquiátricas como la Esquizofrenia (Slachevsky, Pérez, Silva, Orellana, Preñafeta, Alegría \& Peña, 2005) ${ }^{1}$. Dada su mecánica multidimensional, la FV se estudia como un importante núcleo para la evaluación de las funciones cognitivas. El significativo progreso en este campo ha sido factible gracias al avance tecnológico en el estudio de la topografía cortical. Del mismo modo, la FV es materia de interés para la neuropsicología, la neuropsiquiatría, la fonoaudiología y la psicolingüística. Sin embargo, pese a la importancia de su estudio, los diferentes enfoques implicados en cada disciplina a menudo excluyen aspectos relevantes que, a nuestro juicio, mejorarían la comprensión integrada de esta dimensión comunicativa en personas con graves enfermedades mentales y neurológicas. De ahí que nuestro enfoque esté basado en la lingüística clínica funcional y en el análisis del discurso como métodos ideales de análisis de la interacción comunicativa. Para un estudio de estas características, una entrevista clínica siempre será el contexto más ecológico a través del cual se conseguirá obtener datos lingüísticos óptimos.

En el presente estudio analizaremos algunas dimensiones de la FV en personas con diagnóstico de Esquizofrenia de primer episodio como un marcador de déficit lingüístico primario en la enfermedad (Figueroa, Durán \& Oyarzún, 2017). Las variables lingüísticas estudiadas son las pausas extensas, el volumen de voz débil, los titubeos, los falsos inicios y los tartamudeos. A su vez, el objetivo específico es 
establecer la posible relación entre el estadio evolutivo de la enfermedad y los fenómenos discursivos estudiados, con la finalidad de obtener unos marcadores lingüísticos adecuados para el diagnóstico de la enfermedad en estadios tempranos. La hipótesis del estudio sostiene que la pérdida de la FV se origina en el deterioro cognitivo de inicio temprano, impactando las habilidades básicas de la comunicación eficaz desde las primeras etapas de la enfermedad, por ende, la FV puede ser identificada como un marcador informativo de la presencia de la enfermedad (Figueroa, 2015; Figueroa \& Martínez, en revisión). Por lo tanto la hipótesis de partida se basa en aceptar que la FV presenta graves y frecuentes disfunciones que interfieren de modo significativo en el desempeño comunicativo eficiente del hablante afectado desde el primer episodio de la enfermedad. Para tales propósitos se analizaron 50 entrevistas de sujetos chilenos con los siguientes diagnósticos: 25 personas con Esquizofrenia crónica, 25 de primer episodio de Esquizofrenia, pareados por características sociodemográficas con 12 controles sanos.

\section{Marco conceptual}

\subsection{La evaluación de la fluidez verbal}

La FV ha sido estudiada desde la neuropsicología como la habilidad para generar respuestas verbales con ciertos criterios provistos externamente (Turner, 1999). Los paradigmas de evaluación de la fluidez semántica y fonológica, son los más empleados para dar cuenta del funcionamiento neurocognitivo de las personas estudiadas (Huff, 1990; Heaton, Miller, Taylor \& Grant, 2004). Los primeros trabajos corresponden a Thurstone y Thurstone (1938) y Newcombe (1969), que luego fueron sistematizados por Benton (1968). El rigor de estos primeros trabajos permite que en el presente se empleen instrumentos elaborados bajo las mismas premisas de investigación.

La fluidez fonológica se refiere a la capacidad de generar palabras a partir de una restricción fonológica específica, por ej. 'señale la mayor cantidad posible de palabras que inicien con /p/' (Luteijn \& Barelds, 2004). La fluidez semántica, en cambio, evalúa el volumen léxico a partir de una categoría semántica por ej. 'diga nombres de animales' (Benton, 1968). Este enfoque corresponde al campo de estudio de las funciones ejecutivas (FE) que suelen agruparse en torno a las capacidades cognitivas que permiten la planificación, el procesamiento y ejecución de objetivos (TirapuUstárroz, Muñoz-Céspedes \& Pelegrín-Valero, 2002; García, Rodríguez, Martín, Jiménez, Hernández \& Díaz, 2012). Cabe destacar que si se toma en cuenta la perspectiva neuropsicológica para la descripción de la FV, es conveniente revisar algunas condiciones válidas para la evaluación de las FE que juegan un rol central en la constitución funcional de la FV. Según García-Molina, Tirapu-Ustárroz y Roig-Rovira (2007) entre las cuestiones de suma relevancia informativa de las FE, pueden mencionarse las siguientes: 
i. el rol que desempeñan los filtros del control ejecutivo en la manipulación y recuperación de la información almacenada en-línea.

ii. la memoria perceptiva de corto plazo que permite retener la información sensorial;

iii. el control inhibitorio de la interferencia de información irrelevante.

iv. la observación de la relación entre cerebro y conducta con el fin de comprender mejor los procesos subyacentes entre cerebro y funcionamiento cognitivo.

En síntesis, la FV se evalúa principalmente con criterios cuantitativos y una focalización controlada de las FE que se estudian a través de pruebas estandarizadas, verbigracia, las tareas de evocación. Se ha hallado una afectación de ambos tipos de fluidez, fonológica y semántica, en pacientes con Esquizofrenia (Ojeda, Sánchez, Peña, Elizagárate, Yoller, Larumbe, Gutiérrez, Casasis \& Ezcurra, 2010; García et al., 2012; García-Laredo, Maestú, Castellanos, Molina \& Pérez-Moreno, 2015; Brébion, Stephan-Otto, Ochoa, Nieto, Contel \& Usall, 2018). Esta evaluación al ser focalizada en procesos cognitivos independientes, -como es el caso de los sistemas de memoria o, el acceso al léxico- no atiende a fenómenos subyacentes a la interacción comunicativa, ni evalúa en conjunto la serie de procesos neuropsicológicos y lingüísticos que participan en esta actividad cognitiva multinivel. Este será uno de los alcances de este estudio, es decir, poner de manifiesto que las evaluaciones estandarizadas no son lo suficientemente útiles para obtener información relevante desde el punto de vista de la comunicación e interacción. Solo a través de la entrevista clínica médico-paciente, gracias a especialistas en este tipo de entrevistas, se podrá evaluar de una manera más rigurosa la interacción y, por ende, la FV.

Desde la lingüística y en especial, desde la lingüística clínica funcional, la FV corresponde a la habilidad de llenar el tiempo con habla, además de corresponder a la habilidad de hablar en forma coherente, razonada y con frases semánticamente apropiadas al contexto ${ }^{2}$. Asimismo, la FV corresponde a la habilidad de algunas personas de ser creativas en el uso del lenguaje. O bien, es:

"analizar la posibilidad de aislar del fluir del habla espontánea algunos elementos que sean indicativos de los distintos niveles de la producción de habla y que sean medibles" (Goldman-Eisler, 1968: 118).

Consistente con esto, destacamos la observación de Belinchón (1988) al señalar que:

"la fluidez de la realización fonoarticulatoria de los discursos, que facilita, en el interlocutor, la automatización de los procesos implicados en las fases iniciales de la percepción y comprensión del habla". (Belinchón, 1988: 107). 
Desde el punto de vista lingüístico, por tanto, tener FV es dotar al discurso de coherencia temática, es decir, contar un asunto central que aglutine y dé unidad a todo; es, asimismo, dotar al discurso de unidad estructural, o lo que es lo mismo, que todas las partes estén relacionadas entre sí; unidad funcional, que cada parte asuma una función en el todo; es tener unidad lógica o de congruencia semántica para que el conjunto tenga sentido y no se caiga en contradicciones, hecho que les sucede a los sujetos entrevistados; $y$, por ende, es tener relevancia y unidad jerárquica, lo que, todo ello contemplado en un discurso, le confiere inteligibilidad e interpretabilidad como señala Briz $(2008)^{3}$.

Es indudable que al abarcar gran cantidad de funciones cerebrales, la evaluación de la FV con un molde tradicional deja fuera aspectos aclaratorios relevantes de la condición comunicativa del paciente (Lezak 1987; Baldo \& Shimamura, 1998; Schwartz \& Baldo, 2001; Henry \& Crawford 2004; Slachevsky, et al. 2005).

La abundante evidencia acerca de cómo la FV cumple un rol central en la comprensión del discurso y en el cumplimiento de las metas comunicativas, cuya ejecución exitosa está condicionada no solo por la capacidad de mantener la atención focalizada en un propósito comunicativo, sino que además, por la inclusión de las complejas tareas de dicho proceso comprensivo, es decir, depuración, abstracción, interpretación y reelaboración del significado, en suma, interviene en la tarea de la transformación de unidades lingüísticas, en representaciones proposicionales ad hoc en tiempo real (Menjura, 2007; Cuetos, 2011).

Si bien es necesario comprender el funcionamiento de las capacidades cognitivas del paciente, sostenemos que la FV además debe ser observada en el contexto de la interacción como una forma de evaluación complementaria a las tareas tradicionales que suelen aplicarse en la clínica a fin de incorporar las capacidades intersubjetivas. Al considerar el análisis de los errores espontáneos que revelan posibles dificultades en la interacción (Mendizábal, 2004) tenemos la posibilidad de una comprensión global de las funciones y capacidades deterioradas y por sobre todo, de aquellas que aún conserva el afectado.

\subsection{La fluidez como indicador de déficit en la interacción clínica en psiquiatría}

La interacción médico-paciente, en sí misma, constituye un imbricado procesamiento comunicativo que constituye un marco ideal para las pesquisas de algunas dificultades funcionales, junto con la identificación de posibles alteraciones de la cognición presentes en la Esquizofrenia y en otras enfermedades mentales. La Esquizofrenia es una enfermedad mental grave, de curso deteriorante y con una prevalencia del 1\%, sin distinción de clase social, sexo ni etnia. Algunos de sus aspectos centrales están vinculados al deterioro de las funciones cognitivas como las alteraciones en los sistemas de memoria, atención, funciones ejecutivas y el lenguaje 
(Covington, Congzhou, Brown, Naçia, McClain, Fjordbaka, Semple \& Brown, 2005; Barch \& Ceaser, 2012; DSM-V, 2014).

La evaluación de la FV en psiquiatría está presente como un indicador prevalente en escalas muy difundidas de evaluación psiquiátrica como PANSS 4 (Escala para el Síndrome Positivo y Negativo de la Esquizofrenia (PANSS) de Kay, Fiszbein \& Opler, 1987) cuya valoración se expresa en ocho grados: Ausente, Mínima, Leve, Moderada, Severamente Moderada, Severa y Extrema ${ }^{5}$. Paradójicamente, para evaluar los aspectos lingüísticos-comunicativos como la FV, esta escala carece de auténticos criterios lingüísticos, constituyendo una aproximación subjetiva del comportamiento discursivo del paciente. A pesar de estas limitaciones, la entrevista fenomenológica en salud mental plantea una gran ventaja informativa como interacción pues está basada en el vínculo con el paciente y en la exploración de su conducta a través de la semiología psiquiátrica (Chaika, 1974; Labov \& Fanshel, 1977; Chaika \& Lambe, 1985; Chapman \& Chapman, 1987; Belinchón, 1991; Laguna \& Turull, 2000; Vargas, 2003; Covington, et al. 2005; Kuperberg, 2010; Márquez Guerrero, 2010; Salavera \& Puyuelo, 2010).

\section{Metodología}

\subsection{Participantes}

Para este estudio se analizó una muestra de 50 entrevistas del corpus: LEnguaje, PSicosis e Intersubjetividad; LEPSI ${ }^{6}$. Este corpus está constituido por 1200 entrevistas clínicas grabadas y transcritas, realizadas a personas con diferentes diagnósticos psiquiátricos, en los que predomina Esquizofrenia con un 70\%. Para este estudio se seleccionaron 25 entrevistas realizadas a personas con Esquizofrenia crónica (G1-CRE7) y 25 de primer episodio (G2-PEE ${ }^{8}$ ). Para el grupo de primer episodio se consideraron personas con diagnóstico desde hace menos de tres años al momento de la entrevista. Para el grupo de Esquizofrenia crónica se consideraron personas que habían sido diagnosticadas hace más de tres años. El rango de edad de PEE es de entre 14 y 24 años y el de CRE entre 25 y 50 años. El diagnóstico de todos los pacientes incluidos en este corpus y en este estudio fue confirmado por un equipo de psiquiatras, quienes utilizaron la estructura de la entrevista clínica para el DSM-IV (SCID-P, 1997a, 1997b, de First, Gibbon, Spitzer, Williams \& Benjamin (1997)) y la Escala para el Síndrome Positivo y Negativo de la Esquizofrenia (PANSS) (Kay et al. 1987). Todos los pacientes incluidos en este estudio estaban estabilizados psicopatológicamente, recibiendo dosis orales o de depósito de antipsicóticos, por espacio mínimo de un mes. En el caso de los PEE, además estaban insertos en el programa $\mathrm{AUGE}^{9}$. La participación para este estudio contó con un consentimiento informado escrito firmado por los pacientes y un familiar o cuidador responsable, este consentimiento fue aprobado por el comité de ética científica del SSMS ${ }^{10}$ de Santiago. 
Como grupo control, se incluyeron 12 entrevistas del corpus ESECH, Estudio Sociolingüístico del Español de Chile ${ }^{11}$. Estas entrevistas fueron aplicadas a personas sin diagnóstico psiquiátrico, pareadas por edad, sexo y características sociodemográficas, con el resto de los participantes. En la Tabla 1 reseñamos las características de todos los participantes:

Tabla 1. Datos demográfico-clínicos de los participantes.

\begin{tabular}{|c|c|c|c|c|c|c|c|c|c|}
\hline \multirow{2}{*}{ GRUPOS } & \multirow{2}{*}{$\begin{array}{l}\text { Estadio de la } \\
\text { enfermedad }\end{array}$} & \multirow{2}{*}{\begin{tabular}{|l|l|}
\multicolumn{2}{|c|}{ Sexo } \\
$\mathrm{M} H$
\end{tabular}} & \multirow{2}{*}{$\begin{array}{c}\text { Edad } \\
\bar{x}\end{array}$} & \multicolumn{2}{|c|}{$\begin{array}{c}\text { Años de } \\
\text { escolarización }\end{array}$} & \multicolumn{4}{|c|}{ Ocupación } \\
\hline & & & & 8 & 12 & C/Empleo & S/Empleo & $\begin{array}{c}\text { Dueña } \\
\text { de Casa }\end{array}$ & Estudiante \\
\hline G1(G1CRE) & Eqz. crónica & \begin{tabular}{|l|l|}
12 & 13 \\
\end{tabular} & 35 & $100 \%$ & $60 \%$ & $12 \%$ & $48 \%$ & \begin{tabular}{|l|}
$40 \%$ \\
\end{tabular} & $0 \%$ \\
\hline G2(G2PEE) & $\begin{array}{c}\text { Eqz. de primer } \\
\text { episodio }\end{array}$ & 1114 & 18,9 & $100 \%$ & $12 \%$ & $20 \%$ & $48 \%$ & $0 \%$ & $32 \%$ \\
\hline G3(G3CON) & Controles & \begin{tabular}{|l|l|}
6 & 6 \\
\end{tabular} & 33 & $100 \%$ & $66,6 \%$ & $50 \%$ & $8 \%$ & $17 \%$ & $25 \%$ \\
\hline
\end{tabular}

\subsection{Esquema operativo}

El esquema operativo del presente estudio comprendió una selección aleatoria de las entrevistas clínicas del corpus LEPSI. Luego se operativizaron los indicadores de FV estudiados según las observaciones propuestas por Goldman-Eisler (1968). Primero se contabilizó la cantidad de información por unidad de medida que cada participante fue capaz de emitir en la entrevista. En lo que sigue, se analizó la calidad de la información emitida para corroborar la progresión temática para alcanzar el propósito discursivo, que en este caso, correspondía a la explicación o narración acerca del motivo de la consulta u hospitalización del paciente. La información resultante fue sometida al juicio de seis expertos del área de lingüística y psiquiatría, además de una autora (AFB) quienes procedieron a evaluarla en cuanto a calidad y cantidad. Se calculó como coeficiente de concordancia, al cociente entre la sumatoria de las observaciones concordantes y el total de observaciones, el valor de acuerdo interjueces puede desglosarse como sigue: pausas extensas, 85,7\%; turnos de habla con volumen muy débil, 100\%; titubeos, 71,4\%; falsos inicios, 85,7\% y tartamudeos, $85,7 \%$ y el promedio en el cociente de concordancia fue de un 85,7\%. En virtud de la validez y fiabilidad de los criterios evaluados coincidieron en el etiquetado de ausencia o presencia de los indicadores de FV: pausas extensas, turnos de habla con volumen débil ${ }^{12}$ : titubeos, falsos inicios ${ }^{13}$, y tartamudeos que se definen y ejemplifican en la Tabla 2. 249.9 
Tabla 2. Indicadores de fluidez verbal.

\begin{tabular}{|c|c|c|}
\hline INDICADORES & AUSENCIA (2) & PRESENCIA (1) \\
\hline Pausas extensas & \multicolumn{2}{|c|}{$\begin{array}{l}\text { Se considera pausa extendida cuando se produce la interrupción en el flujo de los } \\
\text { turnos de habla. Según los parámetros propuestos por Crockford y Lesser, 1994, se } \\
\text { considera pausa extendida si esta interrupción supera los cinco segundos. }\end{array}$} \\
\hline $\begin{array}{l}\text { Turnos de habla } \\
\text { con volumen muy } \\
\text { débil }\end{array}$ & \multicolumn{2}{|c|}{$\begin{array}{l}\text { Se trata de la dificultad que se genera a partir del bajo volumen de voz utilizado por } \\
\text { el paciente. Las elocuciones se vuelven apenas audibles, los pacientes parecen } \\
\text { musitar sus respuestas, incluso si las condiciones ambientales son óptimas para } \\
\text { escuchar, es decir, una sala con buena acústica. }\end{array}$} \\
\hline Titubeos & \multicolumn{2}{|c|}{$\begin{array}{l}\text { Producción de uno o más sonidos que no tienen relación estructural con la decisión } \\
\text { léxica siguiente, consideramos pausas oralizadas, (ej: “mmm”, “eeeh”) muletillas (ej: } \\
\text { "claro", “o sea", “¿entiende?”, "¿cierto?”) Repeticiones innecesarias de alguna } \\
\text { palabra o frase (ej: “es como si”), alargamientos de sílabas (ej: “aaantes”) }\end{array}$} \\
\hline Falsos inicios & \multicolumn{2}{|c|}{$\begin{array}{l}\text { Interrupción inmediata que afecta al primer fonema, morfema, palabra, o frase. A } \\
\text { menudo se reformula la expresión, aunque el paciente no siempre logra una versión } \\
\text { más informativa. (ej: "por faltar... por falta de... me faltó un remedio") }{ }^{14} \text {. }\end{array}$} \\
\hline Tartamudeos & \multicolumn{2}{|c|}{$\begin{array}{l}\text { Corresponde a la repetición de un fonema, sílaba o parte de una palabra; esta } \\
\text { repetición no cumple ninguna función comunicativa y, generalmente, no forma } \\
\text { parte de la frase u oración (ej: con...con....co...congé...congenia). }\end{array}$} \\
\hline
\end{tabular}

El diseño de este estudio es transversal, comparativo y no experimental. En lo que se refiere a la descripción del procedimiento estadístico utilizado, se realizó un análisis discursivo de las entrevistas transcritas, a partir del cual, se generaron las variables que fueron categorizadas. De esta forma, al tratarse de un estudio con variables cuantitativas múltiples, comprobamos la distribución normal de los datos conseguidos y utilizamos una prueba no paramétrica: 'Chi' cuadrado, que sirvió para correlacionar la presencia o ausencia de las variables pausas extensas, volumen de voz débil, titubeos, falsos inicios y tartamudeos, con el estadio de la enfermedad de los participantes. En todos los casos, el grado de significación estadística se definió en $\mathrm{p}=0.05$. El paquete estadístico utilizado es el SPSS (Statistical Package for the Social Sciences), versión 21 para Windows.

\section{Presentación y análisis de los resultados}

\subsection{Consideraciones previas}

Con el fin de diferenciar el desempeño comunicativo entre el grupo de personas con patología crónica y el de primer episodio, los datos obtenidos están organizados en dos niveles de observación que pueden aportar evidencia convergente: i. en términos descriptivos, según los porcentajes de frecuencia de la variable clínica de estadio de la enfermedad; ii. en términos cualitativos, en relación con la eficacia comunicativa que alcanzaron durante la interacción.

A continuación, presentamos los porcentajes de frecuencia de las variables en estudio y explicitaremos las dos preguntas que sirvieron de base para este trabajo. La primera de ellas es i. ¿existe una relación entre el estadio de la enfermedad y el deterioro de la FV de los sujetos estudiados?; ii.¿existen fenómenos de deterioro de la 
FV específicos del primer episodio de Esquizofrenia? Para las transcripciones de las entrevistas de las que tomamos los ejemplos, hemos utilizado las anotaciones del corpus PERLA de Gallardo Paúls y Valles (2008). ${ }^{15}$

\subsection{Estadio de la enfermedad}

En esta variable clínica fue posible observar diferencias importantes entre los grupos de personas estudiadas. Resultó ser la más informativa respecto del funcionamiento discursivo y fue significativa estadísticamente en 'pausas extensas', 'turnos de habla con volumen débil', 'falsos inicios y tartamudeos', como se resume a continuación en la Tabla 3 y en el Gráfico 1:

Tabla 3. Estadio de la enfermedad e indicadores de fluidez verbal.

\begin{tabular}{|l|c|c|c|c|c|c|c|}
\hline \multicolumn{1}{|c|}{ Variable Clínica } & \multicolumn{7}{|c|}{ Estadio de la enfermedad } \\
\hline \multicolumn{1}{|c|}{ Grupo } & Crónicos & Primer Episodio & Controles & \\
\hline Indicador & $\mathrm{Pr}^{*}$ & $\mathrm{Au}$ & $\mathrm{Pr}$ & $\mathrm{Au}$ & $\mathrm{Pr}$ & $\mathrm{Au}$ & $\mathrm{P}$ \\
\hline Pausas Extensas & 52 & 32,4 & 48 & 35,1 & 0 & 32,4 & $\mathrm{P} 0,006$ \\
\hline Turnos De Habla Con Volumen Débil & 52,2 & 33,3 & 47,8 & 35,9 & 0 & 30,8 & $\mathrm{P} 0,002$ \\
\hline Titubeos & 62,5 & 32,6 & 18,8 & 47,8 & 18,8 & 19,6 & $\mathrm{P} 0,77$ \\
\hline Falsos Inicios & 72,7 & 33,3 & 9,1 & 47,1 & 18,2 & 19,6 & $\mathrm{P} 0,035$ \\
\hline Tartamudeos & 69,2 & 19,4 & 19,2 & 55,6 & 11,5 & 25 & $\mathrm{P} 0,000$ \\
\hline
\end{tabular}

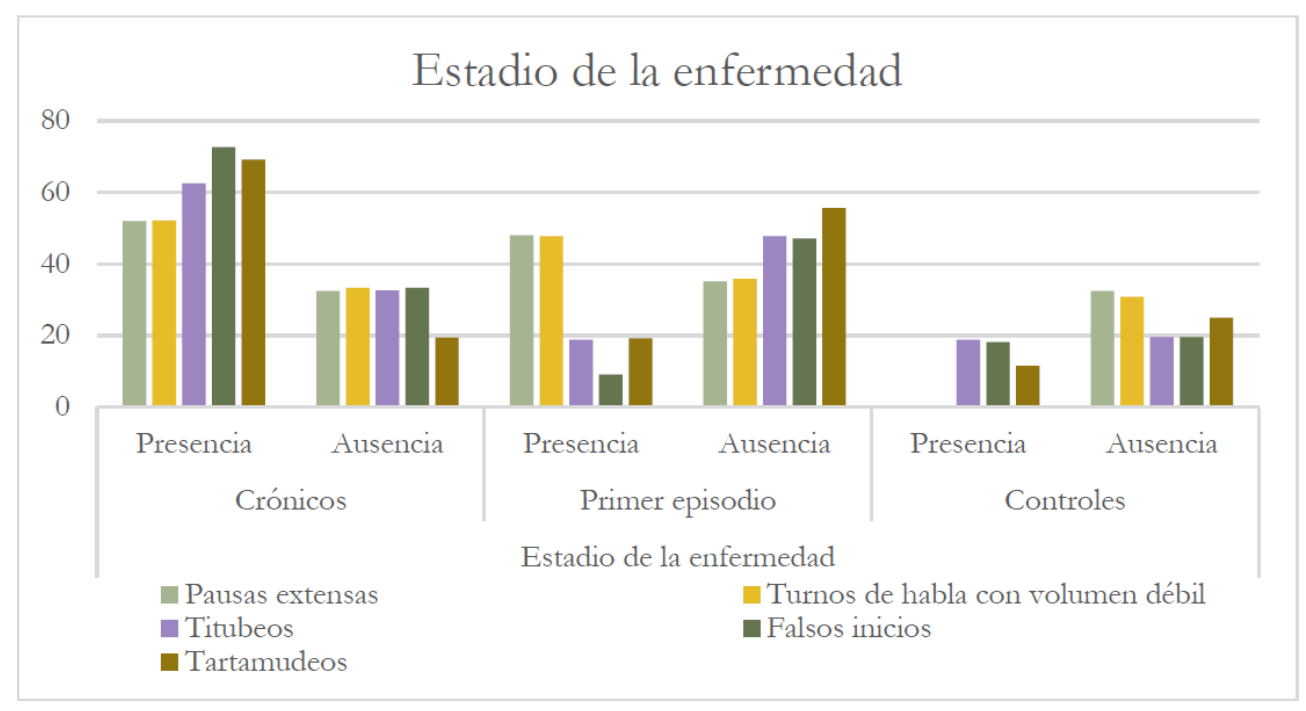

Gráfico 1. Estadio de la enfermedad e indicadores de fluidez verbal.

\subsubsection{Estadio de la enfermedad y pausas extensas}

Las pausas extensas, es decir, aquellas que superan los cinco segundos, se presentaron de manera exclusiva en ambos grupos de Esquizofrenia, como se aprecia en los ejemplos (1) y (2); las pausas extensas sobrevienen fuera de contexto y no responden a tareas o situaciones como recordar información, solucionar un problema 
planteado o, a negarse a responder por desinterés en la conversación o en el tema tratado; por ello constituyen una interferencia que influye de manera categórica en la participación discursiva. Es interesante señalar, en especial en el ejemplo (1), que se inicia con la intervención del entrevistado CRE-H07, que corresponde a una transcripción desde el primer turno de habla, a pesar de que, incluso, expone cuál sería su meta discursiva ('contarles parte de mi vida'), realiza en su discurso una pausa extensa, probablemente por la dificultad de acceso a los datos lingüísticos, propia de la cronicidad. En cambio, en el ejemplo (2) se aprecian dificultades para elaborar una respuesta clara a la pregunta del entrevistador. En la interacción clínica las pausas extensas con frecuencia son provocadas por las dificultades que los pacientes enfrentan al gestionar la información sobre sí mismos. En las personas con Esquizofrenia se ha observado una disminución de su capacidad para contextualizar su experiencia interpersonal, lo que influye en forma directa en que se presente una disociación entre su experiencia y la forma en como la expresan a través del lenguaje (Sass, Pienkos \& Fuchs, 2017). Se encontraron 25 casos de pausas extensas distribuidos en 12 ocurrencias en el grupo de primer episodio, con un $48 \%$ del total y 13 ocurrencias entre los crónicos, es decir un 52\%. El valor de $\mathrm{Chi}^{2}$ es de $10.137^{\mathrm{a}}$, p 0,006 .

Ejemplos:

(1) CRE-H07 001. S: bien/ ¿ah?/ ¿cierto?/ sí// bueno yo me llamo X/ espero que nos llevemos bien hoy día/ para que tengamos un día feliz $<5.0>/$ eh $<5.0>/$ yo quiero decirles mi $<5.0>/$ parte de mi vida// es que puedo darme cuenta de toda la realidad concreta/ que nos hace mejores//cuando nace un hijo //de una pareja//innato/ conciencia innato/ o sea conciencia//y lo otro/ género humano //de la parte congenia/ congenia//congénito/ ¿cierto? // [...]

(2) PEE-H10 001. E: hola/ hola/ ¿cómo está?/ ¿por qué viene X?

002. S: vengo porque// estoy tratando que/ para que/ como que// como que// como que viera que/ no estaría mal/ pero igual/ $\mathrm{mi}$ mamá dice que nunca fue así y yo / ehmmm $<5.0>/$ me gustaría saber si/ eh $<5.0>$ / no sé $<5.0>$ / si creerle o no porque $\mathrm{X} /$ también/ mi primo/ me dijo/ algo como raro/ como que en un momento si/ y después como que no $<5.0>$

003. E: a ver $\mathrm{X} /$ no te entiendo mucho/ quiero que tú me expliques/¿qué es lo que a ti te pasa?

004. S: ///que igual <alarg $>$ me siento mal/ además mi mamá lo niega y yo no sé/ estoy/ creo creer que fue así/ que no hay, no, no estoy seguro, pero yo creo que tal vez fue así. 
Tabla 4. Estadio de la enfermedad y pausas extensas.

\begin{tabular}{|l|l|l|l|l|l|l|l|}
\hline Variable clínica & \multicolumn{7}{c|}{ Estadio de la enfermedad } \\
\hline \multicolumn{1}{|c|}{ Grupo } & \multicolumn{2}{|c|}{ Crónicos } & \multicolumn{2}{c|}{ Primer Episodio } & \multicolumn{2}{c|}{ Controles } & \\
\hline Indicador & Presencia & Ausencia & Presencia & Ausencia & Presencia & Ausencia & $\mathrm{P}$ \\
\hline Pausas Extensas & 52 & 32,4 & 48 & 35,1 & 0 & 32,4 & $\mathrm{P} 0,006$ \\
\hline
\end{tabular}

\section{Pausas extensas}

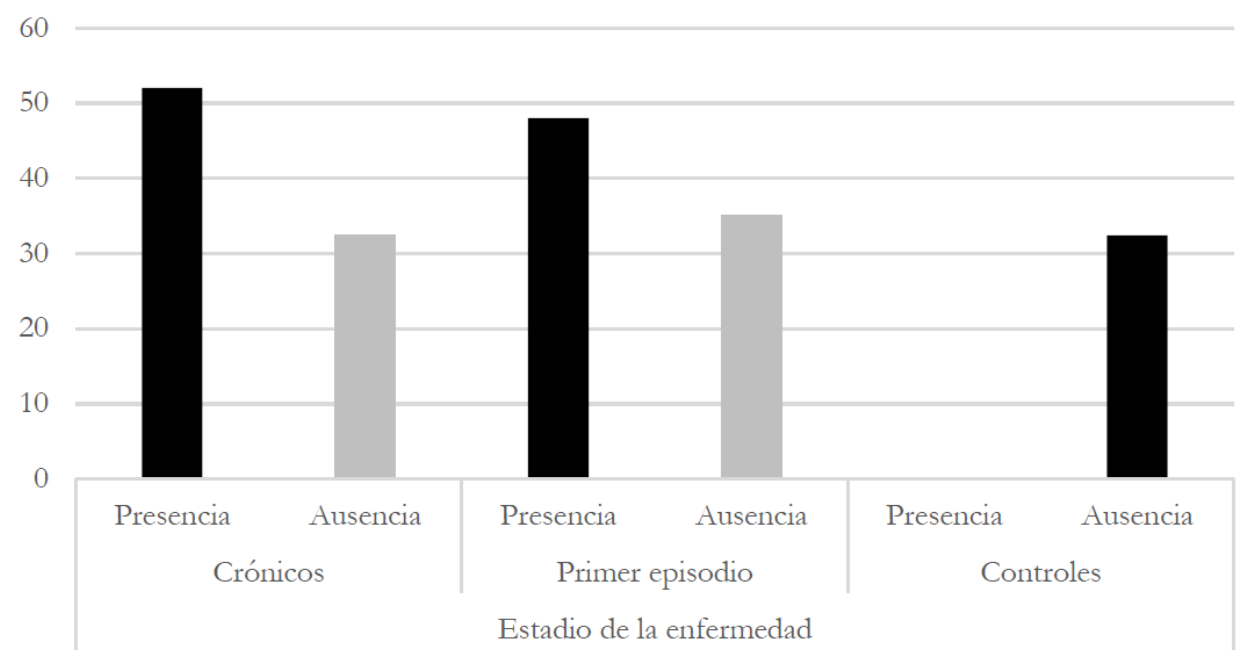

Gráfico 2. Estadio de la enfermedad y pausas extensas.

\subsubsection{Estadio de la enfermedad y turnos de habla con volumen débil}

Este fenómeno no presentó ocurrencias entre los controles. En el primer episodio, se encontraron 11 casos, (47,8\%) y en los crónicos 12 casos, con 52,2\%. Se contabilizaron 23 casos totales, correspondientes al 37\% del corpus total. El valor de $\mathrm{Chi}^{2}$ es de $17,046^{\mathrm{a}}$ y dio como resultado (p, 002), muy significativo como variable. Como podemos observar en el ejemplo (3), hay anotaciones sobre las medidas que debe adoptar el entrevistador para comprender lo dicho, como acercarse al entrevistado y repetir las preguntas cuando no es posible descifrar la respuesta debido al volumen débil.

Ejemplos:

(3) CRE-H10 001. E: don X/¿cómo está usted?

002.S: bien $<^{\circ}()^{\circ}>$

003. E: cuénteme/ ¿por qué estamos acá?

004. S: por tristeza $<^{\circ}()^{\circ}>$ tristeza $<$ aumento de volumen $>$

[entrevistador se acerca más al sujeto] 
005. E: ya/ ¿cómo es eso?/explíqueme

006. S:eeh $<$ pausa or $>/$ me siento triste $<$ alargamiento $>/$ sin ganas $/ / /$ de nada $<5.0>/$ triste $<5.0>/$ [musita para sí, ${ }^{\circ}()^{\circ}$ ] $/$ pero $/ /$ quiero recuperarme/salir adelante.

007. E: ¿cuándo empezó esto? [paciente suspira en un volumen normal] 008. S: ha <pausa or> / hace días/// hace poco/// tenía esa idea fantástica que no puedo recuperar

009. E: ¿cómo?

010. S: al <ininteligible ${ }^{\circ}()^{\circ}>$

011. E: ¿cómo?

012. S: < ininteligible $^{\circ}()^{\circ}>$

(4) PEE-M08 025. E: como por ejemplo/ que sueñes cosas bonitas o desagradables 026. S: no/ sueño cosas bonitas $\left.<^{\circ}()^{\circ}\right\rangle$

027. E: ¿cómo/? habla un poquito más fuerte

029. S: soñar cosas bonitas < volumen normal $>$ /

Tabla 5. Estadio de la enfermedad y turnos de habla con volumen débil.

\begin{tabular}{|c|c|c|c|c|c|c|c|}
\hline Variable clínica & \multicolumn{5}{|c|}{ Estadio de la enfermedad } \\
\hline Grupo & \multicolumn{2}{|c|}{ Crónicos } & Primer Episodio & \multicolumn{2}{c|}{ Controles } & \\
\hline Indicador & Presencia & Ausencia & Presencia & Ausencia & Presencia & Ausencia & Chi $^{2}$ \\
\hline $\begin{array}{c}\text { Turnos De Habla Con Volumen } \\
\text { Muy Débil }\end{array}$ & 52,2 & 33,3 & 47,8 & 35,9 & 0 & 30,8 & $\mathrm{P} 0,002$ \\
\hline
\end{tabular}

\section{Turnos de habla con volumne débil}

60

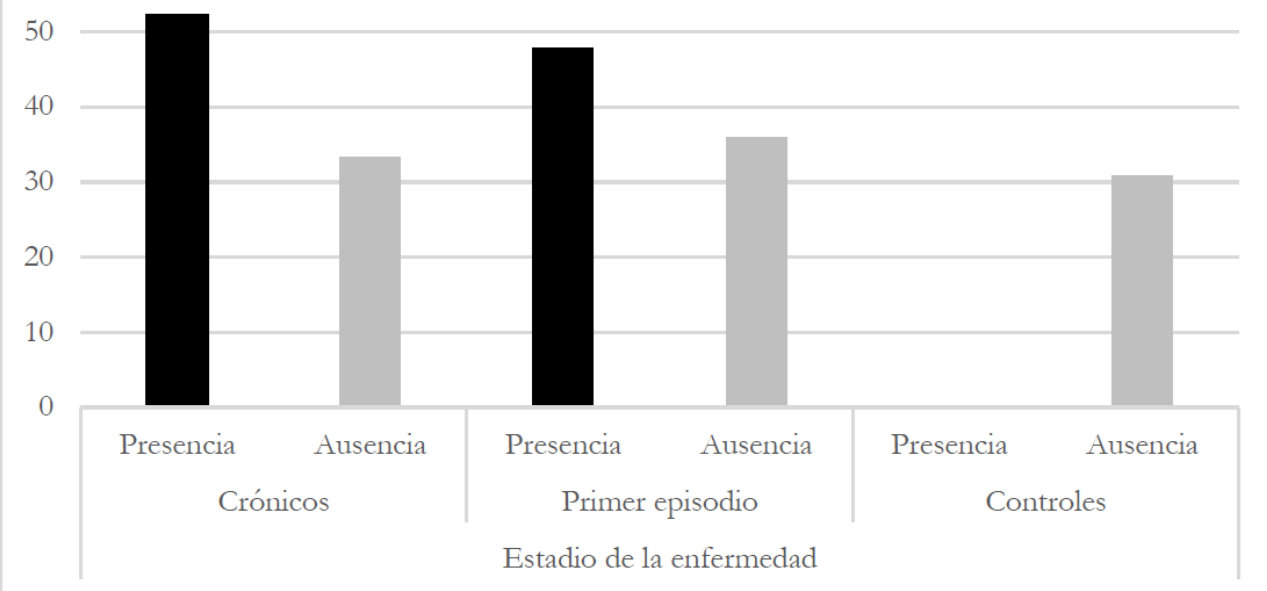

Gráfico 3. Estadio de la enfermedad y turnos de habla con volumen débil. 


\subsubsection{Estadio de la enfermedad y tartamudeos}

Se contabilizaron 26 casos de tartamudeos correspondientes al $41,9 \%$ del total. Entre los controles se contaron 3 casos, (11,5\%), 5 casos entre los de primer episodio, $(19,2 \%)$ y 18 casos entre los crónicos, $(69,2 \%)$, el valor de $\mathrm{Chi}^{2}$ fue de $15.634^{\mathrm{a}}, \mathrm{p}$ 0,000, como se resume en el Gráfico 4 y en la Tabla 6. Las discinesias en Esquizofrenia se presentan con frecuencia y suelen estar asociadas al consumo de antipsicóticos de primera generación. En la actualidad se han masificado los antipsicóticos atípicos que aminoran los efectos extrapiramidales; no obstante, no es raro que aparezcan discinesias tardías en pacientes de larga evolución (Llorca, Chereau, Bayleb \& Lancon, 2002) A pesar de que este fenómeno se dio en forma transversal en los tres grupos de informantes, no presenta las mismas características. Entre los crónicos, como se aprecia en el ejemplo (5) es posible observar que el tartamudeo aparece al inicio del turno provocando una interferencia desde la primera frase. En cambio, entre los sujetos de primer episodio el tartamudeo se suma a las pausas extensas generando una mayor interrupción en el flujo de la información como se aprecia en el ejemplo (6).

(5) CRE-H07 008. E: Bueno/ estábamos hablando de su problema /

009. S: es/ est/ est/ estábamos hablando de mi problema.

010. E: Cuéntenos un poco.

011. S: $\mathrm{Y}<5.0>/$ bueno yo $<5.0>/$ yo / yo / yo/ yo a todo esto conozco todo Chile/ he caminado por todo Chile <pausa or $>$ / y ese día $<5.0>$ / que me gusta a mí/ me gusta fuera de Santiago/ porque no me gusta aquí $<5.0>$ / aquí en Santiago nunca he querido vivir/ también estuve hospitalizado en Putaendo $16<5.0>/$ y mi vida quiero hacerla para allá porque es bonito para allá/ es un pueblito que está ahí en San Felipe/ quiero ir a vivir pa/ pa/ pa/ allá cuando salga de aquí del hospital/ a salir a trabajar todas esas cosas y/ tener una casa allá porque me gustó pa' [para] allá/ yo lo digo sinceramente/ me gustó pa’[para] allá/ yo estuve hospitalizado dos años allá.

(6) PEE-M02 E: ya/ ¿qué significa esto de este señor y la cábala?/ no te entiendo lo que me quieres decir.

S: que esa canción/ algunas partes $<5.0>$ / las palabras/ las tengo como una cábala/ como un amuleto/ como una moneda/ que/ que/que/que/que/que ahí/ahí me/ahí me da los pensamientos/ esos pensam $<5.0>$ / eso es cábala/ como que lo uso como una moneda/ un amuleto/ esas/ para que me dé más suerte.

Tabla 6. Estadio de la enfermedad y tartamudeos.

\begin{tabular}{|c|c|c|c|c|c|c|c|}
\hline $\begin{array}{c}\text { Variable } \\
\text { clínica }\end{array}$ & \multicolumn{7}{|c|}{ Estadio de la enfermedad } \\
\hline Grupo & \multicolumn{2}{|c|}{ Crónicos } & \multicolumn{2}{|c|}{ Primer Episodio } & \multicolumn{2}{c|}{ Controles } & \\
\hline Indicador & Presencia & Ausencia & Presencia & Ausencia & Presencia & Ausencia & $\mathrm{P}$ \\
\hline Tartamudeos & 69,2 & 19,4 & 19,2 & 55,6 & 11,5 & 25 & $\mathrm{P} 0,000$ \\
\hline
\end{tabular}




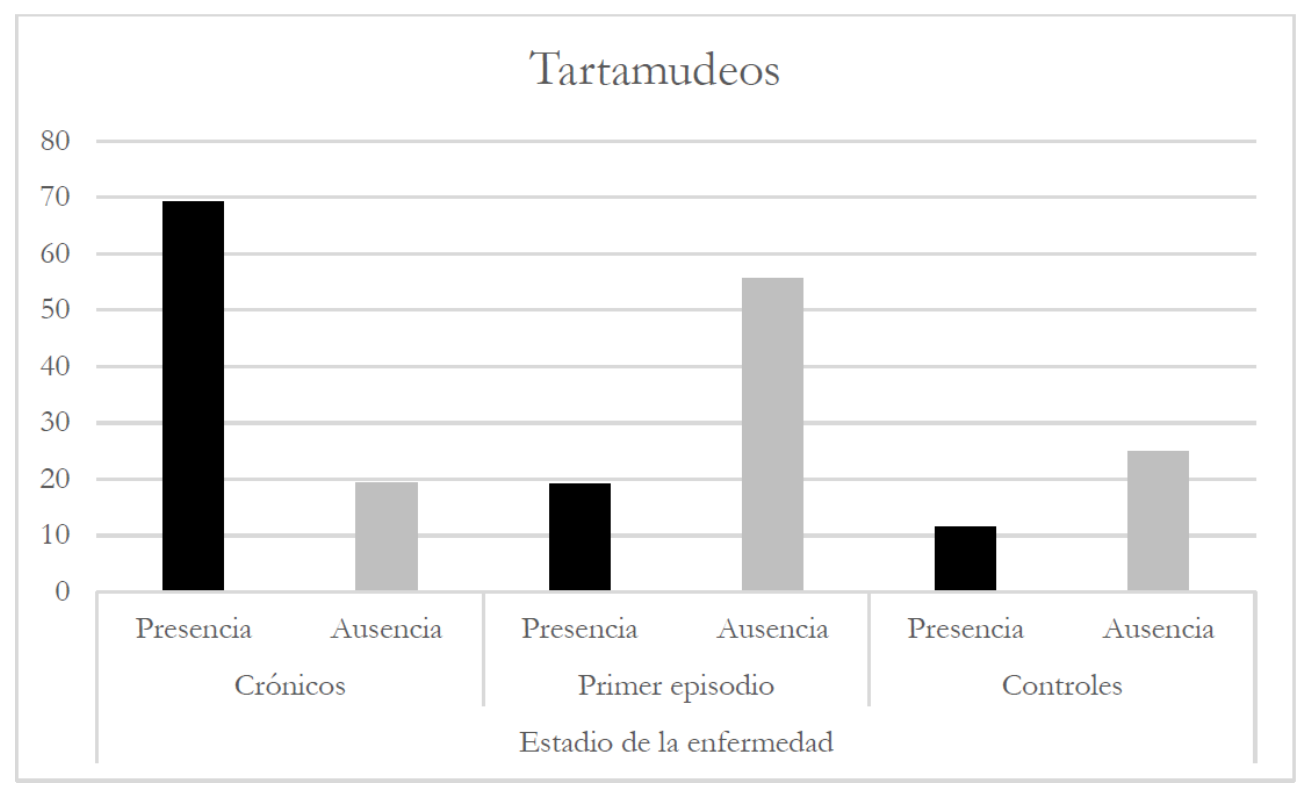

Gráfico 4. Estadio de la enfermedad y tartamudeos.

\subsubsection{Estadio de la enfermedad y falsos inicios}

En este indicador encontramos 2 casos entre los controles, (18,2\%), 1 caso entre los de primer episodio $(9,1 \%)$ y 8 casos entre los crónicos, $(72,7 \%)$. El valor de Chi ${ }^{2}$ fue de $6,727^{a}$ y dio como resultado ( p 0,035), como se aprecia en el ejemplo (7) entre los crónicos observamos las mismas características que las pausas extensas del grupo de primer episodio (8).

Ejemplos:

(7) CRE-H09 005. E: cuéntenos / ¿por qué está aquí?

006. S: bueno//yo estoy//o sea vine// me traj/ [pal cortada] yo estoy aquí por unos problemas/ digamos $/ / /$ eh $/ /$ eh $<5.0>/$ estoy enfermo ahora//esto caducó//mis viejos se fueron de la casa//mi papá se fue de la casa// yo me fui a otra pieza/ con la rubia// estaba entre la rubia y la morena/ y resulta que perdí a la rubia/ poh'[pues] ¿cachai? [obs: cachai= juvenil '¿comprendes?'] / y ahí sentí pena/ mucha pena.

007. E: a ver/¿tuvo un problema con //?/ ¿la eligió//?/ ¿como pareja//?

(8) PEE-M02 023. E: ¿y qué tiene que ver la cantante con el supermercado?/no entiendo//

024. S: eso mismo dicen todos/

025. E: ¿qué te imaginas tú?

026. S: es extra/ ni pe//puede que//que no puedo comprar cosas 
para comer/ porque o si no me voy a convertir en/ $<5.0>$ como ella/ así//como que/ me tinca/ no pa/ que voy a tener los mismos ojos de ella.

Tabla 7. Estadio de la enfermedad y falsos inicios.

\begin{tabular}{|c|c|c|c|c|c|c|c|}
\hline $\begin{array}{c}\text { Variable } \\
\text { clínica }\end{array}$ & \multicolumn{5}{|c|}{ Estadio de la enfermedad } & \\
\hline Grupo & \multicolumn{2}{|c|}{ Crónicos } & \multicolumn{2}{|c|}{ Primer Episodio } & \multicolumn{2}{c|}{ Controles } & \\
\hline Indicador & Presencia & Ausencia & Presencia & Ausencia & Presencia & Ausencia & $\mathrm{P}$ \\
\hline $\begin{array}{c}\text { Falsos } \\
\text { Inicios }\end{array}$ & 72,7 & 33,3 & 9,1 & 47,1 & 18,2 & 19,6 & $\mathrm{P} 0,035$ \\
\hline
\end{tabular}

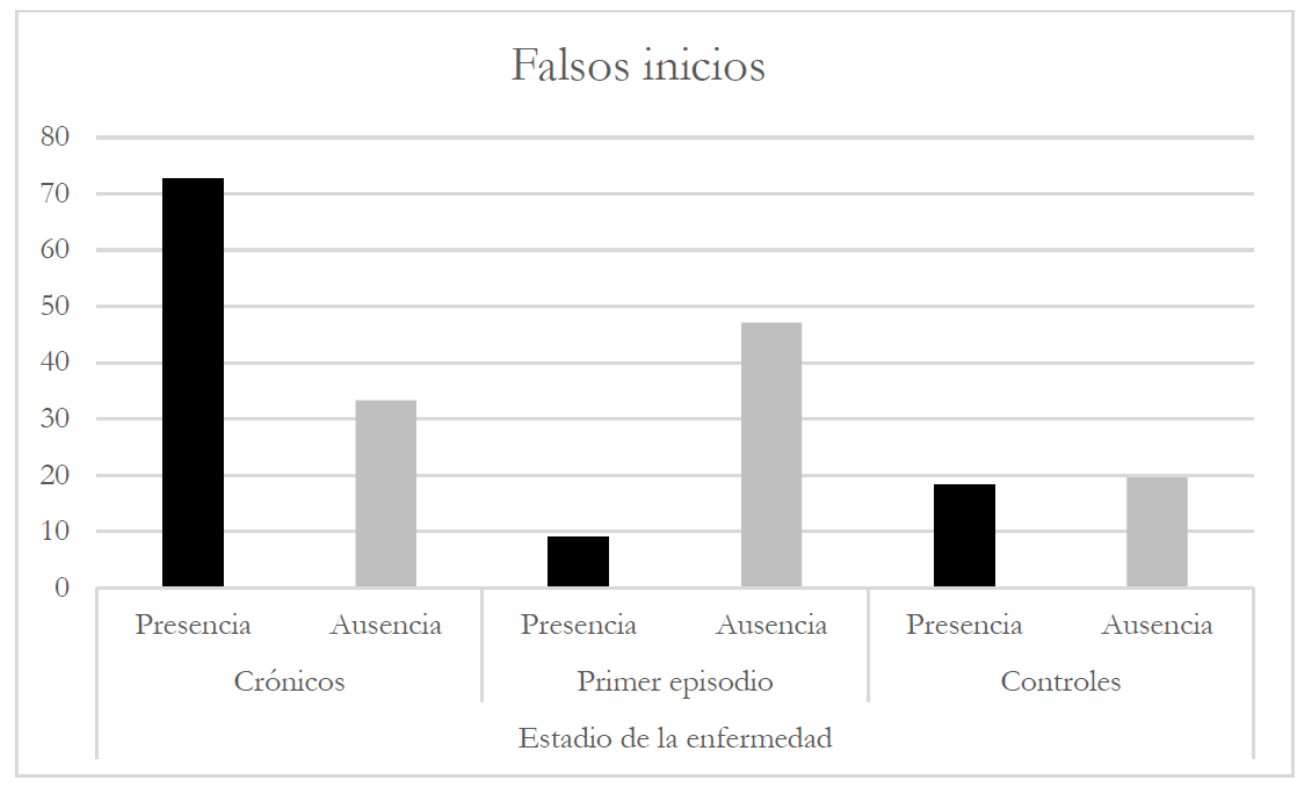

Gráfico 5. Estadio de la enfermedad y falsos inicios.

Los falsos inicios en Esquizofrenia y, en especial en la fase crónica, también pueden estar asociados al deterioro del córtex prefrontal, de las estructuras temporolímbicas y los ganglios basales. Como estructuras cerebrales interconectadas son responsables de los procesos de planificación del discurso, generando una peor ejecución en la elaboración de las frases al deteriorarse (Lawrie, Whalley, Job \& Johnstone, 2003). Asimismo, los hallazgos de alteraciones funcionales de la conectividad medio-prefronto-estriado-talámica en tareas de FV dejan en evidencia el deterioro de estas áreas corticales (Vandevelde, Leroux, Delcroix \& Dollfus, 2017). En cambio, entre la población de primer episodio, se ha argumentado en diversos estudios que esta diferencia puede estar originada en los mecanismos de control inhibitorio y los procesos de automonitoreo que aún se encuentran activos en esta fase 
de la enfermedad, los que incidirían en la actitud dubitativa y errática en la interacción clínica (Figueroa, 2015; Figueroa et al, 2017).

\section{Discusión}

Existe abundante evidencia acerca de la relevancia clínica del déficit hallado en diversos indicadores lingüísticos de FV, tanto en pacientes de primer episodio como Esquizofrenia crónica. Es relevante destacar que viene dada también por la asociación del menor rendimiento en tareas habituales de FV, la gravedad de síntomas psicóticos (Fu, Suckling, Williams, Andrew, Vythelingum \& McGuire, 2005) o la intensidad del déficit cognitivo (Ojeda et al., 2010), así como la influencia de la variable años de educación (García-Laredo et al., 2015) o sexo, habiéndose encontrado patrones diferentes de asociación con variables clínicas y neurocognitivas (Brébion et al., 2018). Finalmente, también los factores genéticos, en particular los antecedentes familiares de la enfermedad, podían afectar el rendimiento de pacientes con Esquizofrenia en pruebas de fluidez verbal (Liang, Deng, Wang, Ma, Li, Brown, Hu, Li, Greenshaw \& Li, 2016). Todas ellas constituyen, pues, líneas futuras de investigación respecto a los indicadores lingüísticos de la FV, que hemos desarrollado en el presente trabajo.

\section{CONCLUSIONES}

A continuación sintetizamos las conclusiones más relevantes del presente estudio.

Concluimos que desde el primer episodio de Esquizofrenia la FV presenta graves y frecuentes disfunciones que interfieren de modo significativo en el desempeño comunicativo eficiente del hablante afectado. En este punto corroboramos la hipótesis de partida en la que se sostiene que la pérdida de la FV se origina en el deterioro cognitivo de inicio temprano, impactando las habilidades básicas de la comunicación eficaz desde las primeras etapas de la enfermedad. De igual manera, corroboramos la hipótesis que señala que la FV puede ser identificada como un marcador informativo de la presencia de la enfermedad.

En relación con la variable clínica, de 'estadio de la enfermedad' resultó ser la más informativa, dado que es la que arrojó mayores diferencias estadísticamente relevantes en el cruce con los fenómenos discursivos estudiados.

Los indicadores de déficit que ostentaron una mayor predictibilidad para primer episodio son los fenómenos 'pausas extensas <5.0>' y 'turnos de habla con volumen débil ${ }^{\circ}()^{\circ}$, en primer lugar, debido a su total ausencia entre los controles del estudio; en segundo término, porque afectan a la estructura discursiva completa dificultando en extremo la interacción, aparentemente, sobrevienen aquí fallas en la planificación de los mensajes del mismo modo que en los pacientes crónicos; en tercer lugar, poseen gran similitud las mismas manifestaciones observadas entre los crónicos y, por 
último, la frecuencia de aparición es significativa entre los informantes del grupo de Esquizofrenia de primer episodio.

Los otros fenómenos pesquisados, 'titubeos', 'falsos inicios' y 'tartamudeos' se encuentran presentes en todos los entrevistados, no obstante, la frecuencia y características que presentan en los tres grupos analizados, genera claras diferencias en la calidad de la eficacia comunicativa de los tres grupos. En los controles, se presentan en una baja frecuencia y, a pesar de que se interrumpe el flujo de información, no se ve afectada la comprensión de lo expresado por el entrevistado. Además, se presentan generalmente en el inicio del turno, como una estrategia de precisión o reformulación de la idea que se está planteando, son predecibles ya que coinciden con aspectos sintácticos, razón por la que podemos asignarles la función de recuperación de información o de estrategias de reparación o reformulación online. En el caso de los crónicos, las ocurrencias de estos fenómenos son abundantes y variables, ya que se presentan no solo en el inicio del turno, sino a lo largo de éste. Con frecuencia, afectan a la comprensión de lo dicho, evidencia que se aprecia en la constante vuelta atrás de los entrevistadores, pidiendo precisiones o realizando una y otra vez preguntas para esclarecer el asunto que ha planteado el entrevistado. En general, la FV involucra un deterioro cognitivo de mayor envergadura en el caso de los crónicos en todos los indicadores discursivos que estudiamos.

En suma, dentro de la gama de alteraciones de diversas funciones del lenguaje en la Esquizofrenia, el estudio de la FV sobresale como un marcador precoz de alta sensibilidad por presentarse ya desde el primer episodio de Esquizofrenia. Asimismo, la enorme demanda atencional que la interacción comunicativa exige ya no puede ser satisfecha por los recursos cognitivos de los afectados, por esta razón las fallas en FV son visibles como un indicador de déficit lingüístico sin necesidad de mediar otro tipo de instrumento estandarizado.

Como posibles proyecciones del estudio, debemos tomar en cuenta el hecho de que son casi inexistentes los trabajos sobre el desempeño comunicativo en primer episodio y en población hispano hablante, por lo que este es, probablemente, un primer acercamiento. Asimismo, surgen algunas interrogantes que será necesario intentar responder en otros estudios posteriores acerca del perfil de la FV en otras psicosis en tareas discursivas similares y su relación estricta con las funciones ejecutivas. También nos parece necesario incorporar las variables sociodemográficas de los hablantes con patología con el fin de correlacionarlas con los fenómenos estudiados, pues se desconoce la realidad ibero-chilena a este respecto. Finalmente, es importante señalar que las alteraciones comunicativas detectadas en este presente trabajo pueden servir de base para propuestas terapéuticas de acompañamiento del tipo: i. compartir con los pacientes los resultados del análisis lingüístico para establecer una conciencia crítica de su situación personal porque se consigue descubrir mecanismos inconscientes; ii. reconstrucción del discurso alienante del sujeto con 
Esquizofrenia recurriendo a grabaciones de sesiones de consulta y a diarios, que son la base del estudio, con el fin de concientizar al paciente sobre el impacto que tiene en el interlocutor y en la interacción fluida. La colaboración con el diseño de herramientas sensibles para implementar diagnóstico clínico precoz de Esquizofrenia es, sin duda, la tarea crucial para favorecer una intervención precoz y específica, y con ello evitar el deterioro cognitivo irreversible.

\section{REFERENCIAS BIBLIOGRÁFICAS}

Baldo, J. V. \& Shimamura, A. P. (1998). Letter and category fluency in patients with frontal lobe lesions. Neuropsychology, 12, 259-267.

Barch, D. M. \& Caesar, A. (2012). Cognition in schizophrenia: Core psychological and neural mechanisms. Trends Cogn Sci. Jan, 16(1), 27-34.

Belinchón, M. (1991). Comunicación y lenguaje en la Esquizofrenia. En C. Castilla del Pino, J. M. Ruiz-Vargas, P. Adarraga, M. Belinchón \& C. Vizcarro (1991), Aspectos cognitivos en la Esquizofrenia (pp. 99-122) Madrid: Trotta.

Benton, A. L. (1968). Differential behavioral effects in frontal lobe disease. Neuropsychologia, 6, 53-60.

Brébion, G., Stephan-Otto, C., Ochoa, S., Nieto, L., Contel, M. \& Usall, J. (2018). Verbal fluency in male and female schizophrenia patients: Different patterns of association with processing speed, working memory span, and clinical symptoms. Neuropsychology, 32(1), 65-76 [en línea]. Disponible en: https://doi.org/10.1037/neu0000394

Briz, A. (2008). Saber hablar. Instituto Cervantes. Madrid: Aguilar.

Chaika, E. (1974). A linguist looks at 'schizophrenic' language. Brain and Language, 1, 257-276.

Chaika, E. \& Lambe, R. (1985). The locus of dysfunction in schizophrenic speech. Schizophrenia Bulletin, 11(1), 8-15.

Chapman, L. J. \& Chapman, J. P. (1987). The search for symptoms predictive of schizophrenia. Schizophrenia Bulletin, 13(3), 497-503.

Covington, M. A., Congzhou H., Brown, C., Naçia, L., McClain, J. T., Fjordbaka, S., Semple, J. \& Brown, J. (2005). Schizophrenia and the structure of language: The linguist's view. Schizophrenia Research, 77, 85-98

Crockford, C. \& Lesser, R. (1994). Assessing functional communication in aphasia: Clinical utility and time demands of three methods. European Journal of Disorders of Communication, 29, 165-182. 
Cuetos, F. (2011). Neurociencia del lenguaje. Bases neurológicas e implicaciones clínicas. Madrid: Panamericana.

DSM-IV (1994). Manual Diagnóstico y Estadístico de los Trastornos Mentales. American Psychiatric Association.

DSM-V (2014). Manual Diagnóstico y Estadístico de los Trastornos Mentales. American Psychiatric Association.

Fernández-Urquiza, M. (2016). Diversidad léxica e informatividad en secuencias de historia producidas por hablantes lesionados de hemisferio de conversaciones semiestructuradas. Pragmalingüistica, 24, 100-121.

Figueroa, A. (2015). Análisis pragmalingüistico de los marcadores de coherencia en el discurso de sujetos con Esquizofrenia crónica y de primer episodio. Tesis doctoral, Universidad de Valladolid, España.

Figueroa, A., Durán, E. \& Oyarzún, S. (2017). La gestión temática como marcador de déficit lingüístico primario en personas con diagnóstico de primer episodio de Esquizofrenia: Un estudio en una muestra chilena. Revista de Lingüistica Teórica y Aplicada, 55(1), 117-147.

Figueroa, A. \& Martínez, C. (en revisión). Las pausas en el discurso de personas con diagnóstico de Esquizofrenia de primer episodio.

First, M. B., Gibbon, M., Spitzer, R. L., Williams, J. B. W. \& Benjamin, L. S. (1997). Structured Clinical Interview for DSM-IV Axis II Personality Disorders (SCID-II). Washington, DC: American Psychiatric Press, Inc.

Fu, C. H. Y., Suckling, J., Williams, S. C. R., Andrew, C. M., Vythelingum, G. N. \& McGuire, P. K. (2005). Effects of psychotic state and task demand on prefrontal function in schizophrenia: An fMRI study of overt verbal fluency. The American Journal of Psychiatry, 162(3), 485-94 [en línea]. Disponible en: https://doi.org/10.1176/appi.ajp.162.3.485

Gallardo Paúls, B. \& Valles, B. (2008). Lingüística en contextos clínicos: La lingüística clínica. Lengua y Habla. Revista del Centro de Investigación y Atención Lingüistica, 12(enero-diciembre), 32-50.

Gallardo, B. \& Moreno, V. (2005). Afasia no fluente. Materiales para su estudio. Vol. 2. Corpus PERLA. Universidad de Valencia. 
García-Laredo, E., Maestú, F., Castellanos, M. Á., Molina, J. D. \& Peréz-Moreno, E. (2015). The relationship between educational years and Phonemic Verbal Fluency (PVF) and Semantic Verbal Fluency (SVF) Tasks in Spanish Patients Diagnosed With schizophrenia, bipolar disorder, and psychotic bipolar disorder. Medicine, 94(39), e 1596 [en línea]. Disponible en: https://doi.org/10.1097/MD.0000000000001596

García-Molina, A., Tirapu-Ustárroz, J. \& Roig-Rovira, T. (2007). Validez ecológica en la exploración de las funciones ejecutivas. Anales de psicología, 23(2), 289-299.

García, E., Rodríguez, C., Martín, R., Jiménez, J. E., Hernández, S. \& Díaz, A. (2012). Test de fluidez verbal: Datos normativos y desarrollo evolutivo en el alumnado de primaria. European Journal of Education and Psychology, 5(1), 53-64.

Goldman-Eisler, F. (1968). Psycholinguistics: Experiments in spontaneous speech. Londres, Nueva York: Academic Press.

Heaton, R. K., Miller, S. W., Taylor, M. J. \& Grant, I. (2004). Revised comprehensive norms for an expanded Halstead-Reitan battery (norms, manual and computer program). Odessa, FL: Psychological Assessment Resources.

Henry, J. D. \& Crawford, J. R. (2004). A meta-analytic review of verbal fluency performance in traumatic brain injured patients. Neuropsychology, 18, 621-628.

Huff, F. J. (1990). Language in normal aging and related neurological diseases. En F. Boller \& J. Grafman (Eds.), Handbook of Neuropsychology (pp. 252-265). Ámsterdam: Elsevier.

Kay, S., Fiszbein, A. \& Opler, L. (1987). The positive and negative syndrome scale (PANSS) for schizophrenia. Schizophrenia Bulletin, 13(2), 261-276 [en línea]. Disponible en: http://www.ncbi.nlm.nih.gov/pubmed/3616518

Kuperberg, G. (2010). Language in schizophrenia Part 1: An introduction language and linguistics compass. Journal Compilation Blackwell Publishing, 4(8), 576-589.

Labov, W. \& Fanshel, D. (1977). Therapeutic discourse: Psichotherapy as conversation. Nueva York: Academic Press.

Laguna, E. \& Turull, N. (2000). Aplicaciones del análisis del discurso en rehabilitación psicosocial con pacientes esquizofrénicos. Revista de Psiquiatría, 27(4), 193-200.

Lawrie, S. M., Whalley, H. C., Job, D. E. \& Johnstone, E. C. (2003). Structural and functional abnormalities of the amygdala in schizophrenia. Send to Ann NY Acad Sci, 985, 445-60. 
Lezak, M. D. (1987). Relationship between personality disorders, social disturbances and physical disability following traumatic brain injury. J Head Trauma Rehabilitation, 2, 57-69.

Liang, S., Deng, W., Wang, Q., Ma, X., Li, M., Brown, M., Hu, X., Li, X., Greenshaw, A. \& Li, T. (2016). Performance of verbal fluency as an endophenotype in patients with familial versus sporadic schizophrenia and their parents. Scientific Reports, 6(1), 32597 [en línea]. Disponible en: https://doi.org/10.1038/srep32597

Llorca, P. M., Chereau, I., Bayleb, F. J. \& Lancon, C. (2002). Discinesias tardías y antipsicóticos: Una revisión. Eur Psychiatry Ed. Esp, 9, 359-369.

Luteijn, F. \& Barelds, D. (2004). GIT-2 Handleiding. Ámsterdam: Harcourt Test Publishers.

Márquez Guerrero, M. (2010). El discurso como síntoma, criterio de pronóstico y elemento terapéutico. En B. Gallardo \& V. Moreno (Ed.), Estudios de Lingüistica clínica. Aplicaciones clínicas (pp. 117-147). Valencia: Universidad de Valencia.

Mendizábal, N. (2004). Los errores espontáneos en la producción lingüística. Artifara: Revista de lenguas y literaturas ibéricas y latinoamericanas, 4.

Menjura, P. (2007). La fluidez discursiva oral una propuesta de evaluación. Ogigia. Revista electrónica de estudios hispánicos, 1, 7-16.

Newcombe, F. (1969). Missle wounds of the brain. A study of psychological deficits. Londres: Oxford University Press.

Ojeda, N., Sánchez, P., Peña, J., Elizagárate, E., Yoller, A. B., Larumbe, J., Gutiérrez M., Casais, L. \& Ezcurra, J. (2010). Verbal fluency in schizophrenia: Does cognitive performance reflect the same underlying mechanisms in patients and healthy controls? The Journal of Nervous and Mental Disease, 198(4), 286-91 [en línea]. Disponible en: https://doi.org/10.1097/NMD.0b013e3181d61748

Sacks, H., Schegloff, E. \& Jefferson, G. (1974). A simplest systematics for the organization of turn-taking for conversation. Language, 50(4), 696-735.

Salavera, C. \& Puyuelo, M. (2010). Aspectos semánticos y pragmáticos en personas con Esquizofrenia. Revista de Logopedia, Foniatría y Audiología, 30(2), 84-93.

San Martín, A. \& Guerrero, S. (2015). Estudio Sociolingüístico del Español de Chile (ESECH): Recogida y estratificación del corpus de Santiago. Boletín de Filología, Tomo L, 1, 221-247. 
Sass, L., Pienkos, E. \& Fuchs, T. (2017). Other worlds: Introduction to the special issue on the EAWE: Examination of Anomalous World Experience. Psychopathology, 50(1), 5-9.

Schwartz, S. \& Baldo, J. (2001). Distinct patterns of word retrieval in right and left frontal lobe patients: A multidimensional perspective. Neuropsychologia, 39, 1209-1217.

Slachevsky, A., Pérez, C. Silva, J., Orellana, G., Preñafeta, M., Alegría, P. \& Peña, M. (2005). Córtex prefrontal y trastornos del comportamiento: Modelos explicativos y métodos de evaluación. Revista Chilena de Neuropsiquiatría, 43(2), 77-170.

Thurstone, L. L. \& Thurstone, T. G. (1938). Primary mental skills. Chicago: Chicago University Press.

Tirapu-Ustárroz, J., Muñoz-Céspedes, J. M. \& Pelegrín-Valero, J. M. (2002). Funciones ejecutivas: Necesidad de una integración conceptual. REV NEUROL, 34(7), 673-685.

Turner, M. A. (1999). Generating novel ideas: Fluency performance in high functioning and learning disabled individuals with autism. Journal of Child Psychology and Psychiatry, 40, 189-201.

Vandevelde, A., Leroux, E., Delcroix, N. \& Dollfus, S. (2017). Fronto-subcortical functional connectivity in patients with schizophrenia and bipolar disorder during a verbal fluency task. The World Journal of Biological Psychiatry : The Official Journal of the World Federation of Societies of Biological Psychiatry, 1-9 [en línea]. Disponible en: https://doi.org/10.1080/15622975.2017.1349339

Vargas, M. (2003). Posibilidades de rehabilitación neurocognitiva en la Esquizofrenia. II Congreso Internacional de Neuropsicología en Internet, Revista de Neurología, 38(5), 473-482.

\section{NOTAS}

1 El presente artículo es parte del proyecto Internacional LEPSI, Lenguaje, Psicosis e Intersubjetividad de la Unidad de Psicolingüística clínica del Depto. de Psiquiatría y Salud Mental Campus Sur de la Facultad de Medicina de la Universidad de Chile y de la Facultad de Medicina de la Universidad de Valladolid, España.

${ }^{2}$ En un estudio sobre diversidad léxica e informatividad en secuencias de lengua producidas por hablantes lesionados de hemisferio derecho durante conversaciones semiestructuradas podemos ver cómo la coherencia se materializa mediante el uso de superestructuras textuales, cuyo correlato psicológico es la noción de esquema cognitivo. Una de las conclusiones de este estudio (Fernández- Urquiza, 2016) es que las secuencias de historia de hablantes lesionados de 
hemisferio derecho son menos informativas que las de los sujetos control, pero que el bajo peso informativo percibido en estos pacientes no es atribuible a una disminución del porcentaje de tipos léxicos. En nuestro caso, el relativo peso informativo viene condicionado por la disminución en la fluidez verbal que ocasionan los síntomas estudiados.

${ }^{3}$ La armonía del periodo sintáctico depende de las construcciones, de la coordinación y de la distribución de todos los miembros en el periodo, así como de la cadencia final. Asimismo, según Briz (2008), el volumen sirve para destacar y realzar los elementos importantes en el discurso y las pausas funcionan como mecanismo de control en el discurso. Cuando estos elementos no son utilizados correctamente, como la velocidad de intervención, el exceso de muletillas, el volumen o los incisos, se convierten en escollos que enlentecen y retardan la exposición oral, provocando la incomprensión por parte de los oyentes.

${ }^{4}$ Escala de síntomas positivos y negativos. Este extracto corresponde a material del Proyecto de apoyo a la evaluación Psicológica Clínica de la Universidad Complutense de Madrid. Este es uno de los ítems que corresponde a los síntomas negativos (N1-N7).

5 N6. FALTA DE ESPONTANEIDAD Y FLUIDEZ DE LA CONVERSACIÓN Reducción de la fluidez normal de conversación asociada con apatía, falta de voluntad, indefensión o déficit cognitivo. Esto se manifiesta por una disminución de fluidez y productividad de la interacción del proceso verbal. Bases de evaluación: Procesos cognitivos verbales observados durante el curso de la entrevista. [PANSS N1-N6]

${ }^{6}$ LEPSI: es un corpus de datos clínicos en psiquiatría, además constituye un proyecto internacional de Este proyecto lo hemos denominado LEPSI (Corpus para el estudio interdisciplinario del lenguaje en la Esquizofrenia y las psicosis de primer episodio), compuesto por dos unidades académicas, pertenecientes a la Universidad de Chile y a la Universidad de Valladolid.

${ }^{7}$ G1-CRE: Grupo 1-crónico de Esquizofrenia

${ }^{8}$ G2-PEE: Grupo 2-primer episodio de Esquizofrenia

9 AUGE: Acceso Universal a Garantías en Salud.

10 SSMS: Servicio de Salud Metropolitano Sur, comité de ética científica, memo 155, 2016.

${ }^{11}$ San Martín y Guerrero (2015).

${ }^{12} \mathrm{La}$ articulación costosa de muchos sujetos con afasias se apoya frecuentemente en el uso de gestos reguladores denominados de 'compás' (Gallardo \& Moreno, 2005) cuya realización simultánea al habla parece ser agente de fluidez discursiva. En el caso de los pacientes que conforman el corpus objeto de este estudio, carecen de este apoyo gestual, pues su dificultad no radica en la articulación propiamente dicha, sino en la organización del pensamiento.

13 En el caso de sujetos con afasia se ha estudiado (Gallardo \& Moreno, 2005) la sobreexplotación de señales paralingüísticas, como la entonación y la longitud silábica como elementos que sirven para verificar que el interlocutor está entendiendo bien su discurso. Esto se traduce en la introducción de pausas dentro del turno, que no llegan a constituir un lugar de transición pertinente (Sacks, Schegloff \& Jefferson, 1974). Es interesante tener en cuenta este lugar de transición pertinente, es decir, "el lugar de la intervención en que resulta posible un cambio de hablante, ya sea por autoselección del hablante 2 o por selección directa realizada por el hablante 1. Como señalan Gallardo y Moreno (2005: 17) "este punto suele coincidir con el final de una unidad sintáctica, lo que supone una mínima pausa demarcativa". En el caso que 
nos ocupa, las pausas de los sujetos con Esquizofrenia, en muchas ocasiones, ni son breves ni coinciden con el lugar de transición pertinente.

${ }^{14}$ Los falsos inicios se pueden relacionar con lo que en sujetos afásicos se estudia como actos de habla tipo tareas de edición y actos activadores de inferencias. Dentro de las tareas de edición podemos destacar los actos de habla borrador, que son los intentos por llegar a la palabra meta. En el caso de los sujetos con Esquizofrenia podemos hablar de falsos inicios o reformulaciones hasta llegar al contenido informativo deseado (Gallardo \& Moreno, 2005).

15 Anotaciones corpus PERLA:

/ Pausa corta, de menos de medio segundo

// Pausa que oscila entre medio segundo y un segundo

/// Pausa de un segundo de duración

(5.0)Pausa extensa de cinco segundos o más

${ }^{\circ}()^{\circ}$ Pronunciación en voz muy baja, próxima al susurro

${ }^{16}$ Hospital Psiquiátrico Dr. Philipe Pinel de Putaendo, V Región, Valparaíso, Chile. 